\title{
Superposition approach for description of electrical conductivity in sheared MWNT/polycarbonate melts
}

\author{
M. Saphiannikova ${ }^{1 *}$, T. Skipa ${ }^{2}$, D. Lellinger ${ }^{2}$, I. Alig $^{2}$, G. Heinrich ${ }^{1,3}$ \\ ${ }^{1}$ Leibniz-Institut für Polymerforschung Dresden e.V., Hohe Straße 6, D-01069 Dresden, Germany \\ ${ }^{2}$ Deutsches Kunststoff Institut, Schlossgartenstraße 6, D-64289 Darmstadt, Germany \\ ${ }^{3}$ Technische Universität Dresden, Institut für Werkstoffwissenschaft, Helmholtzstrasse 7, D-01069 Dresden, Germany
}

Received 10 October 2011; accepted in revised form 20 December 2011

\begin{abstract}
The theoretical description of electrical properties of polymer melts, filled with attractively interacting conductive particles, represents a great challenge. Such filler particles tend to build a network-like structure which is very fragile and can be easily broken in a shear flow with shear rates of about $1 \mathrm{~s}^{-1}$. In this study, measured shear-induced changes in electrical conductivity of polymer composites are described using a superposition approach, in which the filler particles are separated into a highly conductive percolating and low conductive non-percolating phases. The latter is represented by separated well-dispersed filler particles. It is assumed that these phases determine the effective electrical properties of composites through a type of mixing rule involving the phase volume fractions. The conductivity of the percolating phase is described with the help of classical percolation theory, while the conductivity of non-percolating phase is given by the matrix conductivity enhanced by the presence of separate filler particles. The percolation theory is coupled with a kinetic equation for a scalar structural parameter which describes the current state of filler network under particular flow conditions. The superposition approach is applied to transient shear experiments carried out on polycarbonate composites filled with multi-wall carbon nanotubes.
\end{abstract}

Keywords: nanocomposites, multi-wall carbon nanotubes, electrical conductivity, shear flow, modeling

\section{Introduction}

Polymer nanocomposites, filled with attractively interacting conductive anisometric particles such as carbon nanotubes (CNT), graphite nano-platelets or graphenes, are not only in the focus of industrial interest due to numerous possible applications but also draw serious attention from academic research as new functional materials having unique physical properties. So, it is well-known that a minute amount of CNT additive can turn an electrically insulating polymer material into a conductive composite [14]. On the other hand, addition of CNT fillers can lead to a noticeable mechanical reinforcement [5, 6]. The high conductivity in CNT-based composite materials can be ascribed to the formation of the percolation network of interconnected filler particles which may transfer electric current, as well as mechanical stress [7-9]. As discussed in literature $[10,11]$ very small inter-particle distances ( few $\mathrm{nm})$ are needed for low contact resistance and efficient electron transport through the conductive filler network. Therefore, it is not surprising that the percolation network is found to be very fragile, as it can be easily broken under steady shear flow with shear rates of about $1 \mathrm{~s}^{-1}[12,13]$. The shear-induced rupture of filler network represents currently a main obstacle to the widespread introduction of CNTbased polymer nanocomposite products into everyday life. This is because the dominating industrial process for their production is the melt processing

\footnotetext{
${ }^{*}$ Corresponding author, e-mail: grenzer@ipfdd.de
}

(C) BME-PT 
of polymers via extrusion or injection moulding, both processes being characterized by extremely high shear rates. To overcome this impediment, it is necessary to understand and to learn to predict transient electric properties of the sheared polymer nanocomposites. This task is important for industrial applications and very interesting and challenging from the theoretical point of view.

Electrical properties of polymer nanocomposites, filled with attractively interacting conductive anisometric particles, strongly depend on the morphology of filler network $[14,15]$ as well as on the orientation state of individual particles [16, 17]. Therefore, one finds in literature two different approaches for the modelling of the electric properties of those composites. The first approach considers composites in which anisometric conductive particles are randomly distributed in a slightly conducting matrix. Assuming that filler particles do not interact with each other, it is possible to derive the effective electrical conductivity tensor if the second order orientation tensor is known [18]. One of the advantages of this approach is that it naturally accounts for the time evolution of anisotropic conductivity if the time dependence of orientation tensor can be determined (for example by the Folgar-Tucker equation $[19,20])$.

The second approach considers composites in which conductive fillers are randomly distributed (and randomly oriented if anisometric) in a non-conducting matrix. The insulator-to-conductor transition in such systems is induced by the formation of a percolation path, once the filler content has reached a threshold critical value $[21,22]$. Several percolation models generally applicable to isotropic conductive systems have been elaborated: the Swiss Cheese (random void) model and its extensions [23-25], the nodes-links-blobs model [26, 27], Stinchcombe theory on a Bethe lattice [28]. As percolation models are static ones, they are only applicable to homogeneous systems with statistically distributed filler in the equilibrium state. An anisotropic generalization of the Stinchcombe's approach [28], proposed recently by Semeriyanov et al. [29], aims at the description of percolation networks with anisotropic local conductivity represented by resistors with direction-dependent electrical conductivity. Such anisotropy may arise for example from strong shearing of the filler network built from elongated con- ductive particles. Similar to other percolation theories, this newest approach lacks information on time - and shear-dependence of resistor conductivity.

A very promising numerical approach to the electrical conductivity of static and sheared CNT/polymer suspensions has been reported recently by Eken et al. [30,31]. Composite microstructures were generated using a fiber-level simulation method, in which monodisperse fibers (carbon nanotubes) are modeled as a sequence of connected rigid cylinders. The values of electrical conductivity can be determined from generated microstructures using a resistor network algorithm. It has been shown that if a weak shear flow is applied to a viscous fiber suspension, the electric percolation threshold decreases due to shear-induced formation of conductive aggregates [30]. Increasing shear rate has a negative effect on conductive network formation: when shear rate exceeds a critical value the electrical conductivity was found to decrease to the matrix conductivity [31]. For lack of transient percolation theories, we proposed recently a phenomenological approach, in which time and shear-dependence of electrical conductivity is accounted for by coupling percolation theory with an appropriate kinetic equation for buildup and destruction of conductive agglomerates [12, 13]. To our knowledge, agglomeration of nanoparticles in a polymer matrix was first discussed by Schueler et al. [32, 33] for carbon black in uncured epoxy. Heinrich et al. [34] proposed agglomeration of layered nanofillers in a thermoplastic or rubber matrix along with a kinetic equation for description of the time evolution of the shear modulus. In our first simplified approach $[12,13]$ we assumed, that the 'percolation' path is formed by sphere-like conductive agglomerates, containing loosely packed CNTs. These agglomerates can be formed or destroyed in shear flow $[35,36]$. For steady shear conditions constant values for the electrical conductivity and the dynamic shear modulus were found, indicating a stationary state of the filler network due to the competition of shear-induced build up and destruction $[35,36]$. In the quiescent state of melt the destroyed agglomerates were found earlier to be rebuilt in the process of quiescent agglomeration [12-15]. As stated already in [36], for a more realistic description of the filler network and its dependence on the thermo-rheological history a 'superstructure' has to be taken into account, e.g. a 
distribution of agglomerate sizes or a spinodal-type superstructure. In particular, for description of the time-dependent electrical conductivity a hierarchical model [36] assuming different types of agglomerated has been used to fit the experimental data. In the previous studies [36-38] the coupling approach has been tested using either the classical percolation theory [39] or the Generalized Effective Medium (GEM) approximation [40].

Here we present a different superposition approach in which the filler particles are assumed to be separated into percolating and non-percolating phases. In terms of our previous works, the percolating phase can be understood as an 'agglomerate-rich' or as a conductive phase in 'spinodal decomposition'. The conductivity of the percolating phase is described with the help of a coupling approach based on the classic percolation theory. Additionally, we take into account the enhancement of matrix conductivity by the presence of separate filler particles composing the non-percolating phase. For the sake of simplicity, it is assumed that the particles are oriented randomly. This is valid at least for moderate shear rates, less than $10^{3} \mathrm{~s}^{-1}$ (see e.g. $[12,13,41]$ ). The superposition approach is tested on polycarbonate composites filled with multi-walled CNTs at different temperatures. Filler amounts are chosen to be quite close to the percolation threshold, where the effect of shear-induced rupture of filler network is well pronounced [37].

\section{Experimental}

Polycarbonate, Macrolon ${ }^{\circledR}$ type 2600 , was produced by Bayer Material Science AG, Germany. The polymer is of linear structure and amorphous according to the manufacturer specification. The multi-walled carbon nanotubes used in this study were Baytubes ${ }^{\circledR}$ from Bayer Material Science AG with max. 5\% inorganic impurities. The mean outer diameter is of about $13-16 \mathrm{~nm}$ and the tube length varies between 1 and $10 \mu \mathrm{m}$.

The MWNT-polycarbonate samples [37] were provided by Bayer Technology Sevice GmbH Leverkusen, Germany. The compounds were produced by melt-mixing in a twin screw extruder (ZSK 26 MC from Coperion Werner \& Pfleiderer, Stuttgart, Germany) in one processing step. Both, the polycarbonate and the MWNTs were fed into the main feeder of the extruder. After compounding, the MWNT- polycarbonate composites were cooled down in a water bath and were finally granulated with a pelletizer. The granulates were used to prepare diskshaped CNT-polycarbonate plates with a diameter of $80 \mathrm{~mm}$ and a thickness of $2 \mathrm{~mm}$ by injection molding technique at the Thermoplastic Testing Center of Bayer Material Science AG, Leverkusen, Germany. During the injection molding the melt temperature was held at $340^{\circ} \mathrm{C}$, the mold temperature was held at $120^{\circ} \mathrm{C}$. The flow rate during the filling process was set to approximately $5 \mathrm{~cm}^{3} / \mathrm{s}$.

For the conductivity measurements round samples of $25 \mathrm{~mm}$ diameter and of $2 \mathrm{~mm}$ thickness were cut from the mould injected plates. The time-dependent dielectric measurements were performed using a Novocontrol impedance analyser coupled with a laboratory rheometer (Ares, Rheometric Scientific), in which the rheometer plates are equipped with ring electrodes (inner diameter $19 \mathrm{~mm}$, outer diameter $25 \mathrm{~mm}$ ) [12, 13, 35, 36]. The setup with ring electrodes ensures a relatively narrow distribution of the shear rates in the relevant region of electrical field during steady rotation. In the present study a tangential steady shear was applied to the melt by rotating the lower rheometer plate. The electrical conductivity was measured perpendicular to the direction of applied shear. This allowed monitoring of the electrical conductivity in the volume between the two ring electrodes. The conductivity measurements were performed at a single frequency of $1 \mathrm{kHz}$, which is considered to be representative for the DC conductivity [12]. The rheometer is equipped with an oven which maintained stable temperature of the composite melt during the measurements.

\section{Model description}

\subsection{Electrical conductivity}

To describe shear-induced changes in the electrical conductivity of a polymer melt filled with attractively interacting conductive particles, we propose to use a superposition approach similar to that applied by us recently for description of viscoelastic properties of the same nanocomposite system [42]. In the framework of superposition approach we assume that the filler particles can be divided into the percolating and non-percolating phases (see Figure 1).

The percolating phase (network) consists of a group of clusters that are not very far from each other. 


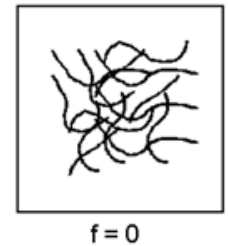

a)

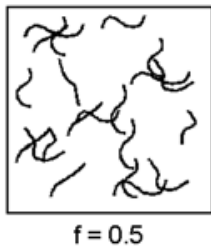

b)

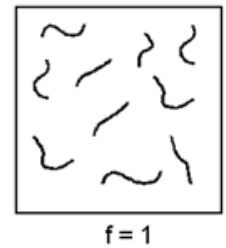

c)
Figure 1. Schematic representation of the time-evolution of filler structure in strong shear flow: (a) initial undestroyed state; (b) intermediate two-phase state, in which the percolating phase is represented by the connected filler clusters and the non-percolating phase by separate well-dispersed particles; (c) final destroyed state, in which all particles are well-dispersed and not connected with one another. Also are shown corresponding values of the parameter $f$, describing the fraction of free nanotubes.

Shear reduces the number of nanotubes in the percolating phase by rupture of nanotubes from the clusters, breaking large clusters into smaller ones and by distributing the clusters evenly in space. All these processes are effectively reducing the fraction of nanotubes that belong to the clusters initially forming a conducting path. The total electrical conductivity, $\sigma$, can be then represented as a sum of two terms (Equation (1)):

$\sigma=X(f) \sigma_{\mathrm{M}}(T)+\sigma_{\mathrm{Net}}(T, f)$

where $\sigma_{\mathrm{M}}(T)$ is the temperature-dependent matrix conductivity and $\sigma_{\mathrm{Net}}(T, f)$ is the conductivity of percolating phase (network); it is equal to zero, if the system is below the electric percolation threshold. A parallel connection of the matrix and percolating network conductivities as described by Equation (1) insures high values of nanocomposite conductivity $\sigma$ above the percolation threshold. Contrary, a connection 'in series' would result in very low values of $\sigma$ comparable with the value of matrix conductivity $\sigma_{\mathrm{M}}$. In Equation (1) $f$ is the fraction of free nanotubes, i.e. of nanotubes not belonging to the percolating phase, and, correspondingly, $1-f$ describes the fraction of nanotubes in the percolating phase.

A quite similar superposition approach to the electrical conductivity of $\mathrm{CNT} /$ polymer composites has been proposed in the studies of Bruck and co-workers $[43,44]$. The total electrical conductivity of MWNT/polystyrene composites in these studies was represented as a sum of two terms: the temper- ature-independent polystyrene conductivity and the conductivity of percolating phase described by a conventional power-law relationship. To model the conductivity increase due to thermal annealing [44], the authors assumed that the effective volume fraction of percolated particles increases with annealing time according to a stretched exponential law, in which the characteristic relaxation time is assumed to obey the Arrhenius temperature dependence. The main difference between the approach of Bruck and co-workers $[43,44]$ and our approach is that the first approach neglects the contribution of non-percolating particles to the total composite conductivity, while we take this contribution explicitly into account by considering a reinforced matrix conductivity described by the first term on the right side of Equation (1). Besides, there is no shear dependence of the effective volume fraction of percolated particles in the first superposition approach, as the authors have only considered the effect of thermal annealing on the composite conductivity [44]. In our study the time evolution of the fraction of percolated nanotubes due to shearing is taken explicitly into account by introducing an appropriate kinetic equation (see section 3.2).

The electric enhancement factor, $X$, in Equation (1) can be calculated for the case of strong contrast, i.e. when the particle conductivity is considerably larger than the polymer conductivity, according to the Equation (2) [18]:

$X(f)=1+\frac{1+3 L}{3 L(1-L)} f \varphi$

Here $\varphi$ is the volume fraction of particles (here CNTs), $f \varphi$ is the volume fraction of free particles and $L=\ln r / r^{2}$ is the depolarization factor defined by the aspect ratio of rigid conductive particle, $r$. Equation (2) has been obtained from a general expression for the effective electrical conductivity tensor (see Equation (12) in ref. [18]) of composites with rod-like conductive inclusions in a slightly conductive matrix. This expression is valid at sufficiently low volume fractions of nanoparticles, when they do not yet percolate. In the case of random orientation distribution of rod-like inclusions one obtains the isotropic conductivity of composite which is proportional to the isotropic matrix conductivity with the coefficient of proportionality given by Equation (2). 
Fitting the viscoelastic data for the same MWNTpolycarbonate composite, we found that the average aspect ratio of the multi-wall carbon nanotubes is about 40 [42]. This value roughly corresponds to the ratio of the Kuhn segment length, reported for the carbon nanotubes to be about $1 \mu \mathrm{m}$ [45], to the nanotube diameter $(13-16 \mathrm{~nm})$. This gives the depolarization factor $L=2 \cdot 10^{-3} \ll 1$, and thus Equation (2) can be reduced to Equation (3):

$X(f)=1+\frac{1}{3 L} f \varphi$

The electrical conductivity of polycarbonate matrix is an ionic type of conductivity and thus strongly increases with temperature. We found that it obeys the Arrhenius law in the region of melt temperatures between 200 and $260^{\circ} \mathrm{C}$ (see Figure 2a) (Equation (4)):

$\sigma_{\mathrm{M}}(T)=\sigma_{\mathrm{M}}^{0} \exp \left(-\frac{E_{\mathrm{a}}}{k_{\mathrm{B}} T}\right)$

with $\sigma_{\mathrm{M}}^{0}=5.78 \cdot 10^{2} \mathrm{~S} / \mathrm{m}$ and $E_{\mathrm{a}}=1.88 \cdot 10^{-19} \mathrm{~J}$. In Equation (4) $T$ is the absolute temperature, $k_{\mathrm{B}}=$ $1.381 \cdot 10^{-23} \mathrm{~J} / \mathrm{K}$ is the Boltzmann constant. This gives $\sigma_{\mathrm{M}}=10^{-9} \mathrm{~S} / \mathrm{m}$ at $230^{\circ} \mathrm{C}$ and $\sigma_{\mathrm{M}}=$ $1.29 \cdot 10^{-7} \mathrm{~S} / \mathrm{m}$ at $340^{\circ} \mathrm{C}$.

The conductivity of percolating network (phase) in Equation (1) is described by a classical percolation equation (Equation (5)):

$\sigma_{\mathrm{Net}}=\left\{\begin{array}{cl}0 & p \leq p_{\mathrm{c}} \\ \sigma_{\mathrm{f}}(T)\left(\frac{p-p_{\mathrm{c}}}{1-p_{\mathrm{c}}}\right)^{\mathrm{t}} & p>p_{\mathrm{c}}\end{array}\right.$

where $p$ is the probability of bond occupation by electrical resistors and $p_{\mathrm{c}}$ is the percolation thresh-

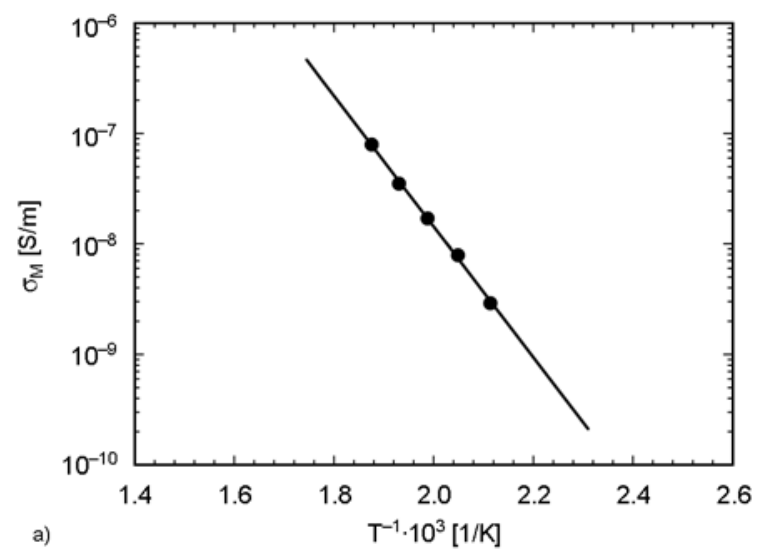

old, at which a cluster (connected group of occupied bonds) spans the whole system. The percolation threshold is a nonuniversal quantity that depends on the space dimension, lattice structure and other microscopic details, e.g. interactions [46, 47]. The conductivity critical exponent $t$ is taken to be equal 2 which is a typical value for three dimensional systems [39]. This value has been found for melt processed MWNT-polycarbonate composites [2]. The conductivity of perfect network, $\sigma_{\text {f }}$, i.e. the value of $\sigma_{\mathrm{Net}}$ at $p=1$ in Equation (5), has been extracted from the equilibrium values of electrical conductivity (i.e. in the absence of shear) for a series of samples with the weight fraction of carbon nanotubes $\Phi=1 \mathrm{wt} \%$. It has been found that $\sigma_{\mathrm{f}}$ is temperature-dependent and obeying as well an Arrhenius law in the region of melt temperatures between 200 and $295^{\circ} \mathrm{C}$ (see Figure 2b) (Equation (6)):

$\sigma_{\mathrm{f}}(T)=\sigma_{\mathrm{f}}^{0}(T) \exp \left(-\frac{E_{\mathrm{a}}}{k_{\mathrm{B}} T}\right)$

with $E_{\mathrm{a}}=6.76 \cdot 10^{-20} \mathrm{~J}$ and $\sigma_{\mathrm{f}}^{0}=5.53 \cdot 10^{7} \mathrm{~S} / \mathrm{m}$. This gives $\sigma_{\mathrm{f}}=3.25 \cdot 10^{3} \mathrm{~S} / \mathrm{m}$ at $230^{\circ} \mathrm{C}$ and $\sigma_{\mathrm{f}}=$ $1.87 \cdot 10^{4} \mathrm{~S} / \mathrm{m}$ at $340^{\circ} \mathrm{C}$. Arrhenius dependence in Equation (6) points on hopping mechanism of electric conductivity.

\subsection{Kinetic equation}

It is assumed that the percolating structure is stabilized by a number of physical bonds that can be broken (or built up) by shearing in a nonlinear regime and can be again re-built during the quiescent time. In our case these physical bonds represent effective attractive interactions between the multi-wall carbon nanotubes. As the total number

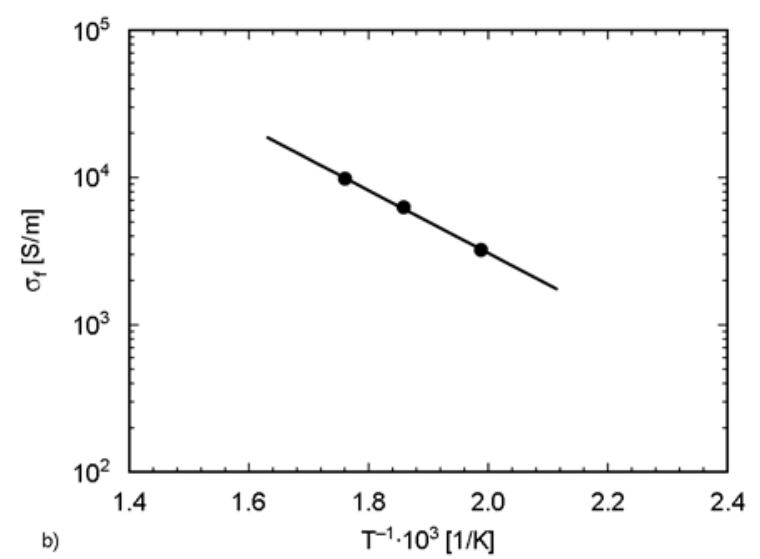

Figure 2. Temperature dependence of the matrix conductivity $\sigma_{\mathrm{M}}(\mathrm{a})$, of the conductivity of perfect network $\sigma_{\mathrm{f}}(\mathrm{b})$. 
of particles - bound ones in the percolating phase and free ones in the non-percolating phase - is not changing, a usual kinetic equation can be written for the change of the fraction of free nanotubes $f$ (Equation (7a)):

$$
\frac{\mathrm{d} f}{\mathrm{~d} t}=a \eta\left(T, \dot{\gamma}_{0}\right) \dot{\gamma}_{0}^{\mathrm{m}}(1-f)-b_{1} \dot{\gamma}_{0}^{\mathrm{n}} f-b_{2} f, \quad 0 \leq f \leq 1
$$

The first (breakage) term on the right side of this equation describes the breakdown of percolation network in a flow with the shear rate $\dot{\gamma}_{0}$, while the second (shear-induced build-up) term takes into account possible shear-induced agglomeration effects [48]. The last (recovery) term in Equation (7a) describes a structure build-up driven by attractive interactions between the multi-wall carbon nanotubes in the absence of shear flow. If originally the nanotubes were well dispersed in the polymer matrix, the process of agglomeration in the absence of shear takes a very long time with the rate constant $b_{2} \sim 10^{-6} \mathrm{~s}^{-1}[35,36]$. The presence of steady shear deformation, as it was found recently by us $[35,36]$, facilitates the structure build-up considerably. For shear rates of $\dot{\gamma}_{0} \leq 0.01 \mathrm{~s}^{-1}$ the values of $b=b_{1} \dot{\gamma}_{0}^{\mathrm{n}}+b_{2} \sim 10^{-3} \mathrm{~s}^{-1}$ were found. Equation (7a) has been tried first to fit the data on stationary and transient electrical conductivity at shear rates up to $1 \mathrm{~s}^{-1}$. It has been found that good fits can be already obtained, if the value $b$ is taken to be shear-rate independent $b \neq b\left(\dot{\gamma}_{0}\right)$. Therefore, in our further considerations we will refer to a simplified version of the kinetic Equation (7a) (Equation (7b)):

$$
\frac{\mathrm{d} f}{\mathrm{~d} t}=a \eta\left(T, \dot{\gamma}_{0}\right) \dot{\gamma}_{0}^{\mathrm{m}}(1-f)-b f, \quad 0 \leq f \leq 1
$$

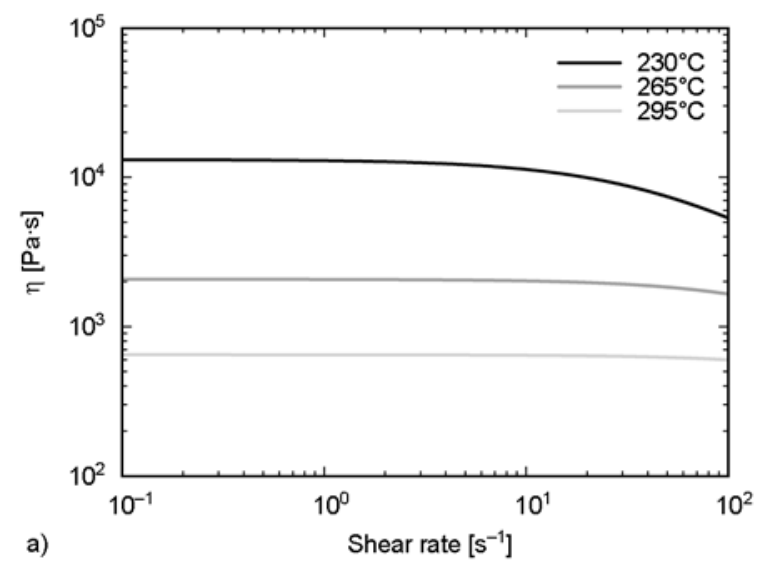

keeping in mind that the effective rate constant $b$ in Equation (7b) describes a joint action of the shear flow and of the attractive interactions between carbon nanotubes on their agglomeration.

Electrical conductivity of the sheared MWNT/polycarbonate melts, as will be shown below, depends strongly on the experimental temperature. This agrees with other studies, in which higher conductivity values have been found for processes involving high temperatures $[15,44,49]$. These correspond to smaller values of the polymer viscosity and thus smaller values of the shear forces acting on the particle aggregates. Assuming the Arrhenius dependence of the zero-shear polyurethane (TPU) viscosity, Bilotti et al. [49] managed to transform time dependences of the CNT/TPU composite conductivities measured at temperatures well above the glass transition temperature on a kind of master curve at times larger than $10 \mathrm{~s}$. The rate of conductivity change was assumed to be inverse proportional to the viscosity of polymer.

To account for the temperature effect in the present study, we propose to introduce into the breakage term on the right side of Equation (7b) a temperature- and shear-dependent matrix viscosity $\eta\left(T, \dot{\gamma}_{0}\right)$ described by the Carreau-WLF model [50] (Equation (8)):

$\eta\left(T, \dot{\gamma}_{0}\right)=\eta_{0} \frac{c_{\mathrm{T}}}{\left(1+c_{\mathrm{T}} t_{1} \dot{\gamma}_{0}\right)^{\mathrm{n}}}$

The polycarbonate $F$ used in this study has $\eta=$ $548 \mathrm{~Pa} \cdot \mathrm{s}, t_{1}=8.8 \cdot 10^{-4} \mathrm{~s}$, the shear-thinning exponent $n=0.79$ and the temperature-dependent parameter $c_{\mathrm{T}}$ given by the Equation (9):

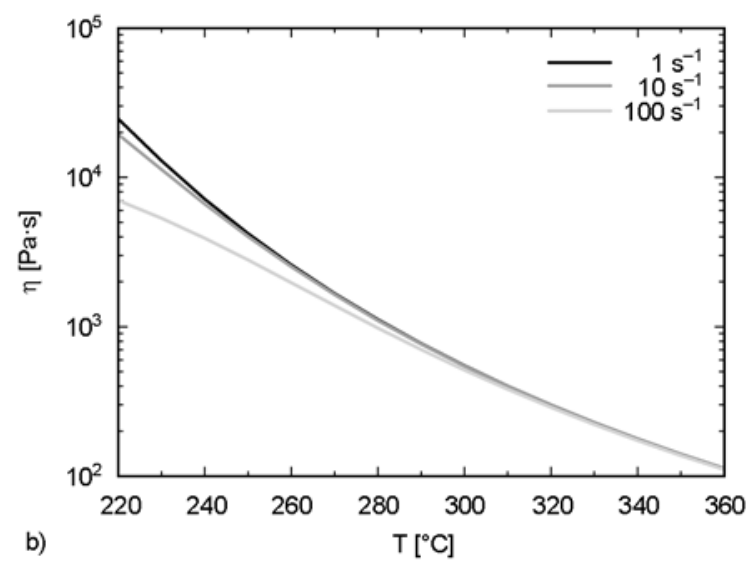

Figure 3. a) Shear dependence of the polycarbonate viscosity at three different temperatures. b) Temperature dependences of the polycarbonate viscosity at three different shear rates. 


$$
\log _{10} c_{\mathrm{T}}=\frac{c_{1}\left(T_{\mathrm{b}}-T_{\mathrm{s}}\right)}{c_{2}+\left(T_{\mathrm{b}}-T_{\mathrm{s}}\right)}-\frac{c_{1}\left(T-T_{\mathrm{s}}\right)}{c_{2}+\left(T-T_{\mathrm{s}}\right)}
$$

where $T_{\mathrm{b}}=300^{\circ} \mathrm{C}, T_{\mathrm{s}}=150^{\circ} \mathrm{C}, c_{1}=8.86$ and $c_{2}=$ $101.6^{\circ} \mathrm{C}$. As can be seen from Figure $3 \mathrm{a}$, the shear viscosity of the polycarbonate melt as described by Equation (8) stays nearly constant for shear rates less than $\dot{\gamma}_{\mathrm{cr}} \sim 10 \mathrm{~s}^{-1}$. This means that at the laboratory conditions, when shear rates do not exceed $1 \mathrm{~s}^{-1}$, one can neglect the shear-thinning effect, $\eta\left(T, \dot{\gamma}_{0}\right)=$ $\eta(T)$. Upon heating the sample from 220 till $320^{\circ} \mathrm{C}$ the polycarbonate viscosity decreases by nearly two orders of magnitude for shear rates below $\dot{\gamma}_{\text {cr }}$ (Figure $3 \mathrm{~b}$ ). At higher shear rates decrease of the viscosity is smaller due to the shear-thinning effect.

\subsection{Coupling part}

To introduce the time and shear dependence into Equation (5) for the electrical conductivity of percolating network, one needs to correlate the occupation probability of network bonds $p$ and the percolation threshold $p_{\mathrm{c}}$ with such material parameters as the fraction of (all) particles and the fraction of free particles. It is clear that in the absence of shear flow the initial occupation probability, $p_{\text {in }}$, should be correlated with the weight fraction of carbon nanotubes, $\Phi$. In our previous rescaling approach, based on the idea of percolation of sphere-like nanotube agglomerates [35-37], the weight fraction of nanotubes has been rescaled to the initial percolation probability, $p_{\mathrm{A}}$, of the agglomerates. In the present study we assume two phases, where the 'conductive phase' undergoes an intrinsic percolation threshold, whereas the non-percolating phase composed from separate nanotubes slightly enhances the matrix conductivity. In terms of our previous works, the percolating phase can be considered as a conductive superstructure formed by the agglomerates (e.g. hierarchy of different agglomerates sizes or 'spinodal structure'). For the sake of simplicity, we will restrict here from the use of rescaling approach assuming instead that the initial occupation probability is equal to the volume fraction of carbon nanotubes, $\varphi$. It can be calculated from the weight fraction $\Phi$ as (Equation (10)):

$$
p_{\text {in }} \equiv \varphi=\frac{\rho_{\mathrm{PC}} \Phi}{\rho_{\mathrm{PC}} \Phi+\rho_{\mathrm{NT}}(1-\Phi)}
$$

In Equation (10) $\rho_{\mathrm{PC}}=1.2 \mathrm{~g} / \mathrm{cm}^{3}$ is the density of polycarbonate and $\rho_{\mathrm{NT}}=2.0 \mathrm{~g} / \mathrm{cm}^{3}$ is the MWNT density. This gives $p_{\text {in }}=0.006$ for $\Phi=1 \mathrm{wt} \%$. This sample as well as the sample with $p_{\text {in }}=0.004(\Phi=$ $0.75 \mathrm{wt} \%$ ) are still highly conductive in the absence of shear. Hence, the percolation threshold for the system under study should lie below 0.004 . If one remembers that the carbon nanotubes are similar to semi-flexible chains, then the percolation threshold can be estimated using the Equation (11) [45]:

$p_{\mathrm{c}} \equiv \varphi_{\mathrm{c}} \sim d^{7 / 5} \cdot l_{\mathrm{p}}^{-3 / 5} L^{-4 / 5}$

where $d \approx 15 \mathrm{~nm}$ is the nanotube diameter, $l_{\mathrm{p}} \sim$ $0.5 \mu \mathrm{m}$ and $L=5 \mu \mathrm{m}$ are nanotube persistence and contour length, correspondingly. Equation (11) provides an estimate of $p_{\mathrm{c}} \sim 0.001$ which will be used in the following.

Now, we are able to couple the electrical conductivity of the percolating phase described by Equation (5) and the kinetic changes in the fraction of free nanotubes given by Equation (7b). Let us first consider the case when the percolating phase is totally destroyed by strong shearing and all nanotubes become free (unconnected). This case corresponds to the values of occupation probability $p=p_{\mathrm{c}}$ and fraction of free particles $f=1$. Contrary, in the case of fully recovered sample all nanotubes belong to the percolating phase: $p=p_{\text {in }}$ and $f=0$. To describe the time- and shear- dependent transition between these two cases, it is natural to introduce a new variable $\Delta p=p-p_{\mathrm{c}}$, that defines a distance from the percolation threshold:

$\Delta p=\Delta p_{\max }(1-f) \quad$ with $\quad \Delta p_{\max }=p_{\text {in }}-p_{\mathrm{c}}$.

Equation (12) together with Equations (1) and (2) implies that initially, in the absence of shear when $f=0$, all carbon nanotubes are in the percolating phase. Hence, the total nanocomposite conductivity is mainly defined by the conductivity of percolating network as in this case the matrix conductivity has a negligible contribution into the total conductivity. Contrary, in the presence of very strong shear when $f \rightarrow 1$ all carbon nanotubes are well dispersed in a polymer matrix and the nanocomposite conductivity is defined by the matrix conductivity slightly enhanced by the presence of conductive particles. 
Using Equation (12), we can rewrite Equation (5) in terms of the shear- or process-dependent parameter $f$ (Equation (13)):

$\sigma_{\mathrm{Net}}=\sigma_{\mathrm{f}}(T) \Delta p_{\max }^{2}\left(\frac{1-f}{1-p_{\mathrm{c}}}\right)^{2}$

Equations (12) and (13) presume that the percolation concentration is proportional to the fraction of 'unfree' $1-f$ particles given by the kinetic Equation (7b). Hence, the percolation probability is not anymore constant but depends on the history of shear application. Other hidden presumptions of present approach are that the particles in the percolation phase are statistically distributed and randomly oriented. The latter presumption is justified by the transmission electron microscopy investigations showing that CNTs agglomerate in the thermoplastic matrix into the sphere-like clusters [13, 15] which can be hardly oriented by the flow. Thus, strong shear first causes destruction of the filler agglomerates which can be possibly followed by an orientation of the individual anisometric filler particles [16]; the latter process is not considered here.

\section{Results and discussion}

In order to investigate how the shear flow affects the electrical conductivity, a number of similar samples were cut out from mould injected plates. All samples were first pre-treated thermo-mechanically (by the procedure reported in [36]) to obtain a welldefined initial state with a high level of electrical conductivity. This initial state corresponds to 'wellagglomerated' multi-wall carbon nanotubes, i.e. the initial value of fraction of free nanotubes, $f_{0}$, is about zero. Different shear rates varying from 0.02

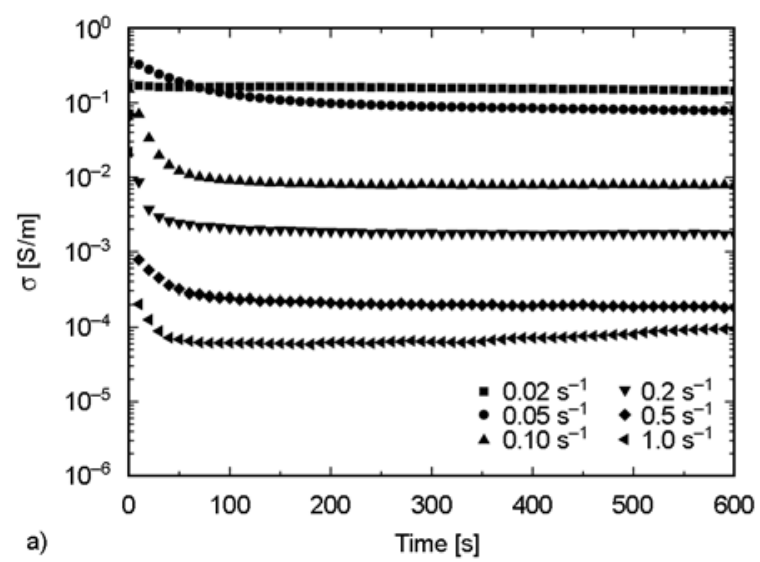

to $1 \mathrm{rad} / \mathrm{s}$ were applied to pre-treated samples. Figure 4a shows typical results measured at $T=265^{\circ} \mathrm{C}$ for a series of samples with $\Phi=0.75 \mathrm{wt} \%$. It can be seen that at all shear rates the electrical conductivity after initial monotonic decrease reaches a steady state value for sufficient duration of the shear deformation (stationary plateau). The steady-state values of electrical conductivity, $\sigma_{\text {st }}$, were taken at $600 \mathrm{~s}$ and then plotted versus shear rate, a corresponding curve is shown in Figure $4 \mathrm{~b}$. As can be seen the electrical conductivity of MWNT/polycarbonate melts exhibits a pronounced 'shear-thinning' effect, similar to that observed for shear viscosity of polymer melts in the nonlinear regime of shearing.

It has been shown by Kharchenko et al. [51] and by us [36] that the shear thinning of electrical conductivity can be fitted by the Carreau equation [52] or a similar Equation (14):

$$
\sigma=\frac{\sigma_{\mathrm{eq}}}{\left(1+t_{1} \dot{\gamma}_{0}\right)^{\mathrm{m}_{\sigma}}}
$$

where $\sigma_{\mathrm{eq}}$ is the equilibrium value of electrical conductivity, $m_{\sigma}$ is the shear-thinning conductivity exponent and $t_{1}$ is the time constant. The shear thinning exponents for conductivity were found to be 0.66 [51] and 2 [36]. However, only one series of samples with a certain wt $\%$ of MWNTs and at one chosen melt temperature has been measured in the previous studies. So, it is not clear a priory, whether the shear-thinning curves measured for different weight fractions and at different temperatures are characterized by different values of the shear-shinning conductivity exponent or a single one. If the latter case is true, it will open an opportunity to predict the electrical conductivity at diverse processing

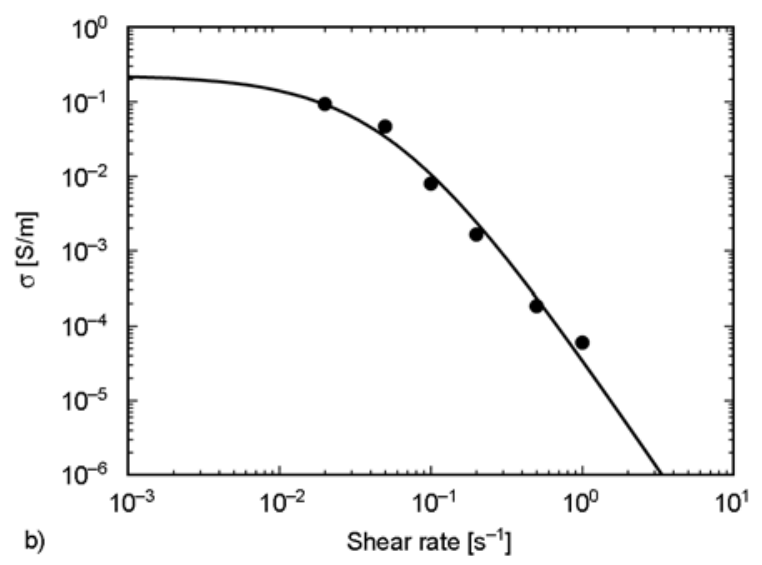

Figure 4. a) Time dependent electrical conductivity at six different shear rates: $0.02,0.05,0.10 .2,0.5$, and $1.0 \mathrm{~s}^{-1}$. $\Phi=$ $0.75 \mathrm{wt} \%, T=265^{\circ} \mathrm{C}$. b) Dependence of stationary electrical conductivity on shear rate. $\Phi=0.75 \mathrm{wt} \%, T=$ $265^{\circ} \mathrm{C}$. Line represents a fit with the help of Equation (14), the shear-thinning exponent $d=3$. 
conditions (after extrusion or injection moulding) based on results measured under laboratory conditions. In the following subsection we shall investigate such a possibility, which is supported by experiments varying both temperature and shear rate.

\subsection{Steady-state electrical conductivity}

The steady-state electrical conductivity can be calculated from Equation (13) by putting into it the stationary fraction of nanotubes in the percolating network (Equation (15)):

$$
1-f_{\mathrm{st}}=\frac{1}{1+a b^{-1} \eta\left(T, \dot{\gamma}_{0}\right) \dot{\gamma}_{0}^{\mathrm{m}}}
$$

Equation (15) is given by a stationary solution of Equation (7b) when $\mathrm{d} f / \mathrm{d} t=0$. Thus,

$$
\sigma_{\mathrm{Net}}^{\text {st }}=\frac{\sigma_{\mathrm{f}}(T) \Delta p_{\max }^{2}}{\left[1+a b^{-1} \eta\left(T, \dot{\gamma}_{0}\right) \dot{\gamma}_{0}^{\mathrm{m}}\right]^{2}}
$$

Equation (16) contains three parameters: shearthinning conductivity exponent, $m_{\sigma}=2 m$, the rup-
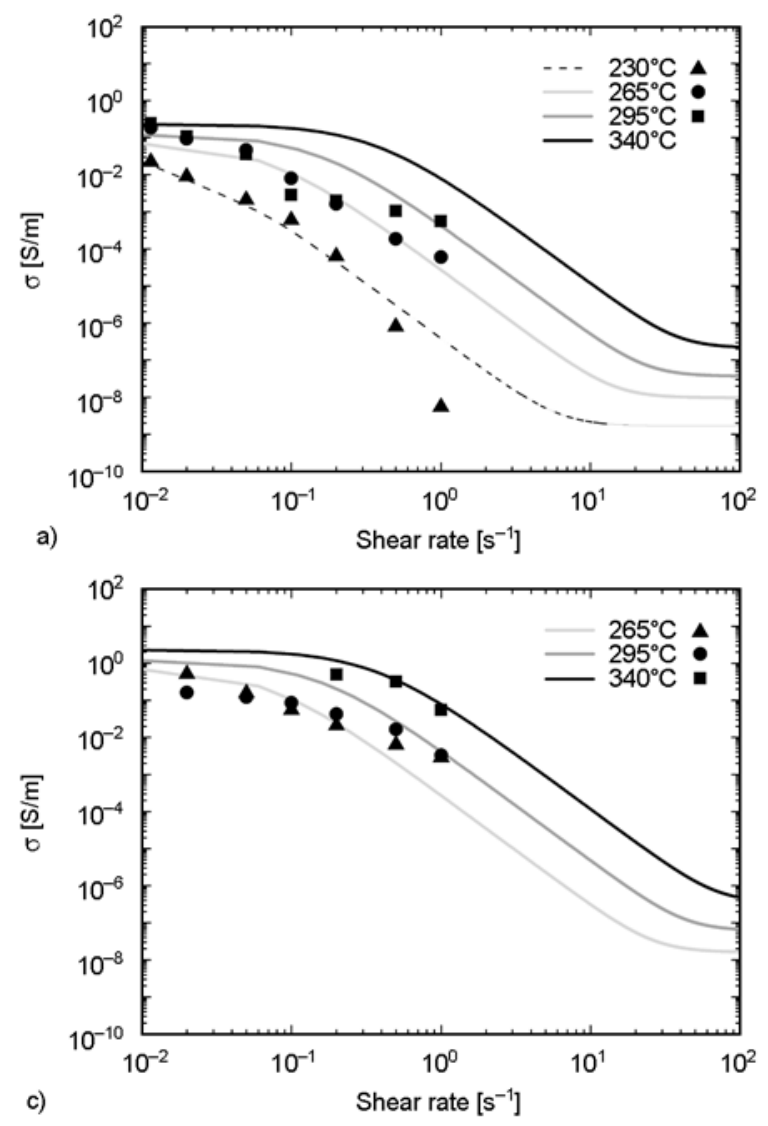

ture strength $a$ and the rate constant $b$; the latter two appear in combination as a product $t_{\mathrm{f}}=a b^{-1}$.

Figures $5 \mathrm{a}$ and $5 \mathrm{~b}$, filled symbols, show shear-thinning dependences of the electrical conductivity measured for polymer composites at two MWNT concentrations ( 0.75 and $1 \mathrm{wt} \%)$ and three melt temperatures $\left(230,265\right.$ and $\left.295^{\circ} \mathrm{C}\right)$. The strongest shear-thinning effect has been observed for the system with the lowest MWNT concentration $0.75 \mathrm{wt} \%$ and at the lowest temperature $230^{\circ} \mathrm{C}$. The steadystate values of the electrical conductivity of the sample sheared at $1 \mathrm{~s}^{-1}$ falls 6 orders of magnitude compared to the unsheared system (Figure 5a). The reason of such dramatic behaviour is that, on the one hand, this system is very close to the insulatorconductor transition, and on the other hand, it has the highest matrix viscosity of $10 \mathrm{kPa} \cdot \mathrm{s}$. Hence, very high shear stresses easily bring a weakly percolating MWNT network into a well-dispersed state, characterized by a very low value of the electrical conductivity close to that of the polycarbonate
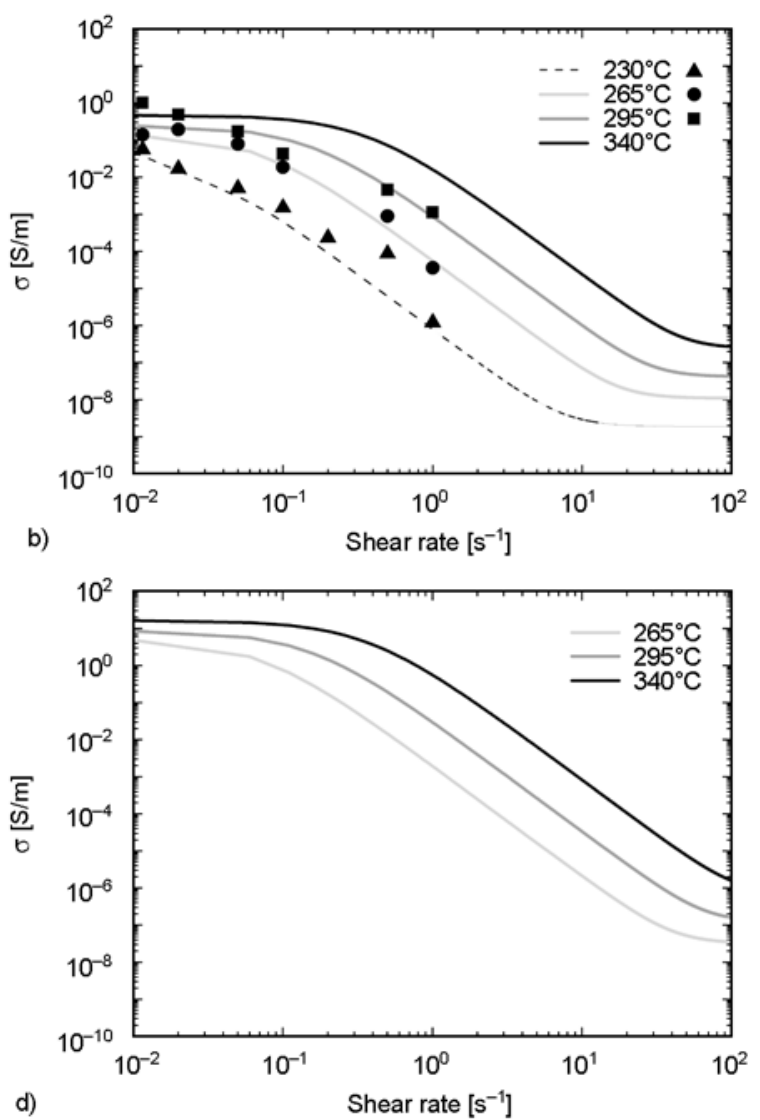

Figure 5. Stationary conductivity at different temperatures and shear rates for the systems with different weight fractions of MWNTs: $0.75 \mathrm{wt} \%$ (a), $1 \mathrm{wt} \%$ (b), $2 \mathrm{wt} \%$ (c) and $5 \mathrm{wt} \%$ (d). Experimental data - symbols, fitting - lines. The first symbols plotted at the shear rate of $0.0115 \mathrm{~s}^{-1}$ correspond to the conductivity values measured for the unsheared systems. In figures (a) and (b) black solid curve shows predictions for $T=340^{\circ} \mathrm{C}$. 
matrix. As the matrix viscosity (and thus the shear stress) rapidly decreases with temperature, the shearthinning effect has been found less pronounced for the samples with the same MWNT concentration but sheared at higher temperatures. For example, at $295^{\circ} \mathrm{C}$ the electrical conductivity decreases only two orders of magnitude both for the system with 0.75 and $1 \mathrm{wt} \%$ MWNTs. However, the system with higher MWNT concentration exhibits a smaller shear-thinning of the electrical conductivity (it falls only 4 orders of magnitude) being farther from the percolation threshold (Figure 5b).

All these features can be captured in the frame of proposed superposition model which describes the conductivity of a percolation network with a changing number of electrical resistors. The fitting results for the systems with $0.75,1$ and $2 \mathrm{wt} \%$ MWNTs are presented on Figure 5a-c by different lines. All data are fitted with the same fitting parameters: the shear thinning exponent $m_{\sigma}=3$ and $t_{\mathrm{f}}=0.025$. Thus, the shear-thinning exponent of electrical conductivity is found to be considerably higher than it was reported in two previous studies [36, 51]. At laboratory conditions it was possible to measure credible data for the systems with 0.75 and $1 \mathrm{wt} \%$ MWNTs only below $300^{\circ} \mathrm{C}$, since above this temperature the samples start to leak from the rheometer cell due to lowered viscosity. Fortunately, after verification of the model on samples with low MWNT concentrations and temperatures it is possible to extrapolate the theory predictions onto the systems with higher MWNT concentrations and temperatures, i.e. to the conditions relevant for nanocomposite processing. Theoretical predictions at the temperature of $340^{\circ} \mathrm{C}$, which was found to be optimal for the injection moulding of MWNT/polycarbonate melts [42], are shown on the same Figures $5 \mathrm{a}$ and $5 \mathrm{~b}$ as a black solid line. Additionally, on Figure $5 \mathrm{~d}$ we present the model predictions for the system with $5 \mathrm{wt} \%$ MWNTs at three melt temperatures (265, 295 and $340^{\circ} \mathrm{C}$ ). One can see that the theory predicts a strong reduction of electrical conductivity even for this highly filled system at all melt temperatures, provided that the shear rates exceed $10^{2} \mathrm{~s}^{-1}$.

\subsection{Percolation curves}

There is no general accepted picture in literature what happens with the electrical percolation threshold in sheared systems. While the present authors interpret considerably lower values of conductivity in the sheared system as a reduction of the volume content of the agglomerates composing the conductive percolation network [35-37], other authors attribute the reduction of conductivity to a shift of the percolation threshold to higher values [53, 54]. There even exists an opinion that percolation threshold can become anisotropic under application of the shear forces, the possibility considered in the frame of anisotropic percolation theory, in which the occupation probability is different in two perpendicular directions [55]. Theory of anisotropic electrical conductivity developed by Semeriyanov et al. on a Bethe lattice [29] does not presume an anisotropic percolation threshold but an anisotropic conductivity of electrical resistors which may differ in directions parallel and perpendicular to the shear direction. The percolation threshold stays unaffected by the shearing and is solely defined by a functionality of the Bethe lattice. The latter should be adjusted using experimental data for a particular nanocomposite system as was shown in Subsection 3.3. The model proposed in this study is based on the same presumption of shear-independent percolation threshold and neglect orientation effects.

Figure 6a shows theoretical predictions for percolation curves at the fixed value of the percolation threshold $p_{\mathrm{c}}=0.005$ but at increasing shear rates: 0 , $0.1,1.0$ and $10 \mathrm{~s}^{-1}$. Compared to the curve calculated at the quiescent conditions (in the absence of shear), the electrical conductivity falls by two orders of magnitude at $\dot{\gamma}_{0}=0.1 \mathrm{~s}^{-1}$, five orders of magnitude at $\dot{\gamma}_{0}=1 \mathrm{~s}^{-1}$ and eight orders of magnitude $\dot{\gamma}_{0}=10 \mathrm{~s}^{-1}$, becoming comparable with the matrix conductivity. Such behaviour, especially the form of two last curves, may give a false impression that the percolation threshold shifts to higher values with the increase of shear rate. From the data in Figure $6 \mathrm{~b}$ one may conclude that the conductivity data measured for the annealed (equilibrated) sample at the absence of shear and for the injection molded sample can be fitted in the frame of superposition approach without assuming the shift of percolation threshold. However, we do not exclude that for some other systems with strong orientational effects (for example, low viscous epoxies filled with CNTs [53]) the percolation threshold will indeed shift to higher values under strong shearing. This has been found also for CNTs in high viscous polycarbonate 

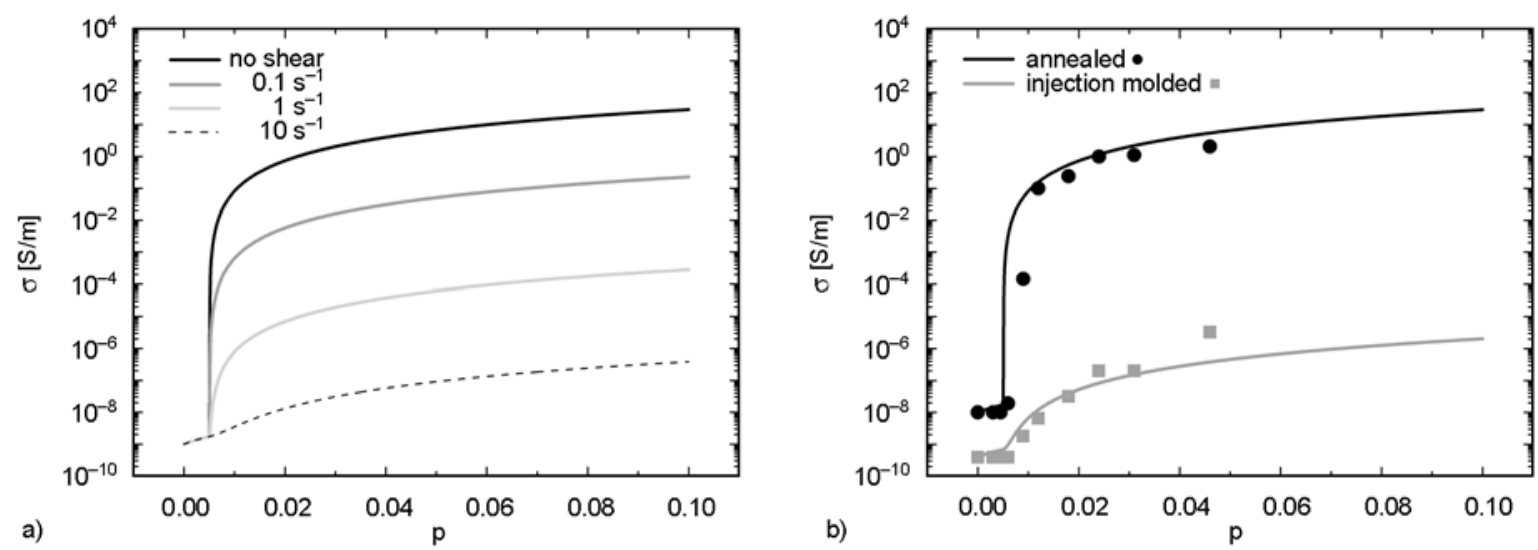

Figure 6. a) Theory prediction for percolation curves at the fixed value of the percolation threshold $p_{\mathrm{c}}=0.005$, but at increasing shear rates: $0,0.1,1.0$ and $10 \mathrm{~s}^{-1} . T=230^{\circ} \mathrm{C}$. b) Fit for two samples (lines): Annealed sample is measured at $T=230^{\circ} \mathrm{C}$ in the absence of shear (circles). Injection molded sample $\left(T=340^{\circ} \mathrm{C}\right.$, average shear rate $10.0 \mathrm{~s}^{-1}$ ) is measured at $T_{\text {room }}$ (squares).

melt for very high shear rates typical for microinjection moulding [41].

\subsection{Transient electrical conductivity}

Electrical conductivity of industrially relevant products produced by the melt processing via extrusion or mould injection depends on a whole history of a product preparation. Therefore, it is important to learn to predict not only stationary values of the electrical conductivity but also its transient behaviour in a wide range of shear rates. For the superposition approach proposed here, a correct prediction of transient behaviour means determination of two parameters in the kinetic Equation (7b): the rupture strength $a$ and the rate constant $b$. Fitting of the shear-thinning curves (Section 4.1) only provides information about the ratio of these two parameters: $t_{\mathrm{f}} \equiv a / b=0.025$. One can separate parameters $a$ and $b$ by fitting the time-dependent data for a number of polymer nanocomposite systems with different filler loadings and at different temperatures. To lend the superposition approach a predictive quality at very high shear rates, it is essential to keep both parameters constant for a whole data pool, although it may result in higher discrepancies between experimental data and model predictions at particular shear rates. All data discussed further are fitted with the same values of fitting parameters: $a=$ $0.25 \cdot 10^{-4} \mathrm{~Pa}^{-1} \cdot \mathrm{s}^{-1 / 2}$ and $b=10^{2} \mathrm{~s}^{-1}$.

Comparison of experimental data and model predictions for the system with $0.75 \mathrm{wt} \%$ MWNTs is presented in Figure 7 for two different temperatures: $230^{\circ} \mathrm{C}$ (Figure $7 \mathrm{a}$ ) and $265^{\circ} \mathrm{C}$ (Figure $7 \mathrm{~b}$ ). As can be seen, there is some variation in the initial values of electrical conductivity between different curves. Therefore, we have to introduce a correction on the initial state into our model by adjusting an initial value of the fraction of free nanotubes. This considerably improves the fits of time-dependent data measured at the laboratory conditions. The theory predictions at $230^{\circ} \mathrm{C}$ agree rather well with the experimental data at shear rates up to $0.1 \mathrm{~s}^{-1}$, while at higher shear rates the theory overestimates the rate of shear-induced breakage. The transient behaviour at the shear rate of $1 \mathrm{~s}^{-1}$ is badly predicted as the stationary value for this curve is considerably overestimated in the frame of our model (see also Figure 5a). Otherwise, the theory predicts really good the time-dependent data measured at $265^{\circ} \mathrm{C}$ (Figure $7 b$ ).

Comparison of experimental data and model predictions for the system with $1 \mathrm{wt} \%$ MWNTs reveals a similar tendency: there is a noticeable overestimation of the rate breakage at $230^{\circ} \mathrm{C}$ (Figure $7 \mathrm{c}$ ), while at $265^{\circ} \mathrm{C}$ the theory predictions fit the data rather good (Figure $7 \mathrm{~d}$ ). Contrary, at $295^{\circ} \mathrm{C}$ the theory slightly underestimates the rate of shear-induced breakage (not shown here). Finally we show on Figures $7 \mathrm{e}$ and $7 \mathrm{f}$ comparison between the measured data and the model predictions for the system with $2 \mathrm{wt} \%$ MWNTs. The scarceness of data at $340^{\circ} \mathrm{C}$ (Figure 7f) is due to the problem of sample leaking caused by the lowered matrix viscosity. Nevertheless, the principle agreement between the experiment and theory even at this elevated temperature is rather good. 

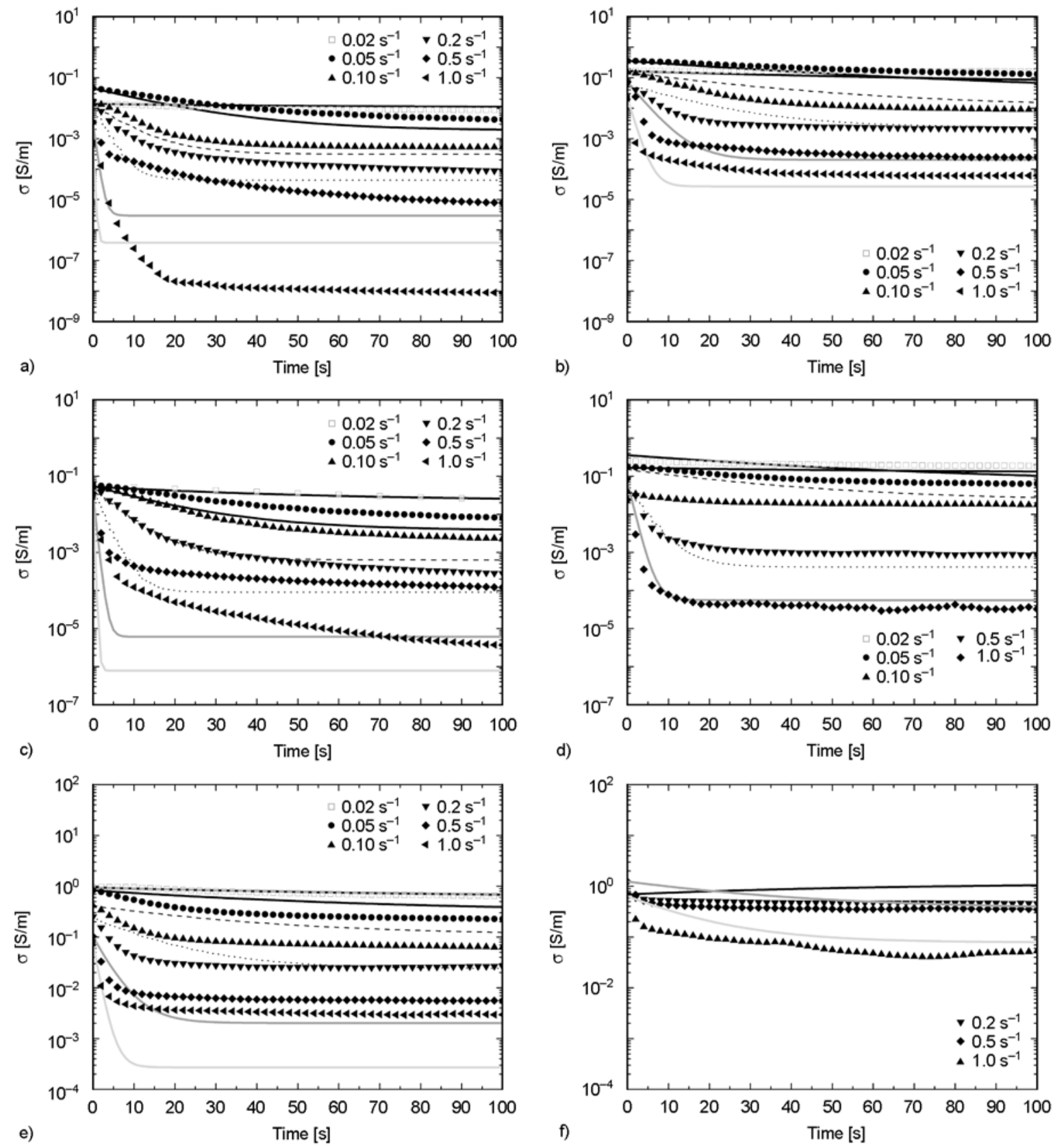

Figure 7. Time dependent electric conductivity at different temperatures and shear rates for the systems with different weight fractions of MWNTs: a) $\Phi=0.75 \mathrm{wt} \%, T=230^{\circ} \mathrm{C}$; b) $\Phi=0.75 \mathrm{wt} \%, T=265^{\circ} \mathrm{C}$; c) $\Phi=1 \mathrm{wt} \%, T=$ $230^{\circ} \mathrm{C}$; d) $\Phi=1 \mathrm{wt} \%, T=265^{\circ} \mathrm{C}$; e) $\Phi=2 \mathrm{wt} \%, T=265^{\circ} \mathrm{C}$; f) $\Phi=2 \mathrm{wt} \%, T=340^{\circ} \mathrm{C}$. Experimental data - symbols, fitting - lines.

Presently, we use a simple form of kinetic equation given by expression (7b) which contains only two terms and, hence, three fitting parameters (including the shear-thinning exponent). To improve description of the time-dependent data, one needs to consider a more elaborated kinetic equation, which may contain an additional term for shear-induced aggregation, or even a hierarchic set of kinetic equations [36]. However, this will introduce a number of additional fitting parameters and thus consid- erably complicate the task of their extraction from the fitting procedure. This will be a topic of the next paper, while in the present studies we maintained the simplified picture of filler network kinetics.

\section{Conclusions}

In this paper we present a superposition approach for description of electrical conductivity in sheared polymer melts filled with attractively interacting conductive (anisometric) particles. The main 
assumption of superposition approach is that the filler particles can be separated into two phases: the percolating one and non-percolating one. In the absence of shear, if the percolation probability exceeds the percolation threshold, (nearly) all particles belong to the percolating phase (network) and strongly contribute to the electrical conductivity. In the presence of shear, some particles are ruptured from the network (or the percolating phase is dispersed into smaller parts) and thus they are considered to belong to the non-percolating phase. The process of network rupture, i.e. how large is the fraction of free particles, is described by an appropriate kinetic equation. This fraction defines the enhancement of electrical conductivity of the polymer matrix given by the theory of effective electrical conductivity of composites with spheroidal inclusions at low volume fractions [18]. Presently, for the sake of simplicity, it is assumed that the particles belonging to the non-percolating phase are oriented randomly. This seems to hold for small and medium shear rates. Though it is possible to account for orientational effects in the non-percolating phase using for example the Folgar-Tucker equation $[19,20]$.

The conductivity of percolating phase is described with the help of a coupling approach based on the classic percolation theory. There are two main assumptions of our approach:

1) the percolation probability depends on the history of shear application,

2) the particles in the percolation phase are oriented randomly.

If for some other system it will be find out that the percolating clusters are noticeably deformed by the flow before their breakage, one can try to account for this effect using the theory of anisotropic electrical conductivity proposed recently by Semeriyanov et al [29].

The superposition approach has been tested in the present studies on the polycarbonate composites filled with multi-walled CNTs. Four different melt temperatures, three nanotube concentrations and up to six different shear rates have been used to extract the time-dependent and shear-thinning behaviour of electrical conductivity. This allowed us to show that the 'shear-thinning' exponent of electrical conductivity is about 3 which is considerably higher than it was reported in two previous studies [36, 51]. In overall, we found a rather good agreement between the theory predictions and the experimental data. This means that results measured at relatively small shear rates in the laboratory conditions can be extrapolated to much higher shear rates typical for extrusion and injection moulding conditions. Thus, the superposition approach, if implemented in a commercial flow software, may open a way to predict - at least semi-quantitatively - the electrical conductivity of industrial products produced from polymer composites filled with highly attractive conductive particles at diverse processing conditions.

\section{Acknowledgements}

This work was carried out with the support of the BMBF Project 'CarboNet' No. 03X0504E and 03X0504F. Dr. Bierdel (Bayer Technology Services GmbH, Leverkusen, Germany) is greatly acknowledged for providing the samples for rheoelectrical measurements. Further, we would like to thank Dr. Fyodor Semeriyanov (IPF Dresden) for very helpful discussions concerning the modelling part.

\section{References}

[1] Coleman J. N., Curran S., Dalton A. B., Davey A. P., McCarthy B., Blau W., Barklie R. C.: Percolationdominated conductivity in a conjugated-polymer-carbon-nanotube composite. Physical Review B, 58, 74927495 (1998).

DOI: 10.1103/PhysRevB.58.R7492

[2] Pötschke P., Dudkin S. M., Alig I.: Dielectric spectroscopy on melt processed polycarbonate-multiwalled carbon nanotube composites. Polymer, 44, 5023-5030 (2003).

DOI: 10.1016/S0032-3861(03)00451-8

[3] Sandler J. K. W., Kirk J. E., Kinloch I. A., Shaffer M. S. P., Windle A. H.: Ultra-low electrical percolation threshold in carbon-nanotube-epoxy composites. Polymer, 44, 5893-5899 (2003).

DOI: 10.1016/S0032-3861(03)00539-1

[4] Bauhofer W., Kovacs J. Z.: A review and analysis of electrical percolation in carbon nanotube polymer composites. Composites Science and Technology, 69, 1486-1498 (2009).

DOI: $10.1016 /$ j.compscitech.2008.06.018

[5] Pötschke P., Fornes T. D., Paul D. R.: Rheological behavior of multiwalled carbon nanotube/polycarbonate composites. Polymer, 43, 3247-3255 (2002).

DOI: $10.1016 / \mathrm{S} 0032-3861(02) 00151-9$

[6] Pötschke P., Abdel-Goad M., Alig I., Dudkin S., Lellinger D.: Rheological and dielectrical characterization of melt mixed polycarbonate-multiwalled carbon nanotube composites. Polymer, 45, 8863-8870 (2004).

DOI: $10.1016 /$ j.polymer.2004.10.040 
[7] Heinrich G., Klüppel M.: Recent advances in the theory of filler networking in elastomers. Advances in Polymer Science, 160, 1-44 (2002).

[8] Fan Z., Advani S. G.: Rheology of multiwall carbon nanotube suspensions. Journal of Rheology, 51, 585604 (2007). DOI: $10.1122 / 1.2736424$

[9] Pegel S., Pötschke P., Petzold G., Alig I., Dudkin S. M., Lellinger D.: Dispersion, agglomeration, and network formation of multiwalled carbon nanotubes in polycarbonate melts. Polymer, 49, 974-984 (2008). DOI: $10.1016 /$ j.polymer.2007.12.024

[10] Gojny F. H., Wichmann M. H. G., Fiedler B., Schulte $\mathrm{K}$.: Influence of different carbon nanotubes on the mechanical properties of epoxy matrix composites - A comparative study. Composites Science and Technology, 65, 2300-2313 (2005).

DOI: $10.1016 /$ j.compscitech.2005.04.021

[11] Gojny F. H., Wichmann M. H. G., Fiedler B., Kinloch I. A., Bauhofer W., Windle A. H., Schulte K.: Evaluation and identification of electrical and thermal conduction mechanisms in carbon nanotube/epoxy composites. Polymer, 47, 2036-2045 (2006).

DOI: $10.1016 /$ j.polymer.2006.01.029

[12] Alig I., Skipa T., Lellinger D., Pötschke P.: Destruction and formation of a carbon nanotube network in polymer melts: Rheology and conductivity spectroscopy. Polymer, 49, 3524-3532 (2008).

DOI: $10.1016 /$ j.polymer.2008.05.037

[13] Alig I., Skipa T., Engel M., Lellinger D., Pegel S., Pötschke P.: Electrical conductivity recovery in carbon nanotube-polymer composites after transient shear. Physica Status Solidi B, 244, 4223-4226 (2007).

DOI: $10.1002 / p s s b .200776138$

[14] Alig I., Lellinger D., Dudkin S. M., Pötschke P.: Conductivity spectroscopy on melt processed polypropylene-multiwalled carbon nanotube composites: Recovery after shear and crystallization. Polymer, 48, 1020-1029 (2007).

DOI: 10.1016/j.polymer.2006.12.035

[15] Alig I., Lellinger D., Engel M., Skipa T., Pötschke P.: Destruction and formation of a conductive carbon nanotube network in polymer melts: In-line experiments. Polymer, 49, 1902-1909 (2008).

DOI: $10.1016 /$ j.polymer.2008.01.073

[16] Villmow T., Pegel S., Pötschke P., Wagenknecht U.: Influence of injection molding parameters on the electrical resistivity of polycarbonate filled with multiwalled carbon nanotubes. Composites Science and Technology, 68, 777-789 (2008).

DOI: 10.1016/j.compscitech.2007.08.031

[17] Du F., Fischer J. E., Winey K. I.: Effect of nanotube alignment on percolation conductivity in carbon nanotube/polymer composites. Physical Review B, 72, 121404/1-121404/4 (2005).

DOI: 10.1103/PhysRevB.72.121404
[18] Zheng X., Forest M. G., Lipton R., Zhou R., Wang Q.: Exact scaling laws for electrical conductivity properties of nematic polymer nanocomposite monodomains. Advanced Functional Materials, 15, 627-638 (2005). DOI: $10.1002 / \mathrm{adfm} .200400200$

[19] Lertwimolnun W., Vergnes B., Ausias G., Carreau P. J.: Stress overshoots of organoclay nanocomposites in transient shear flow. Journal of Non-Newtonian Fluid Mechanics, 141, 167-179 (2007).

DOI: $10.1016 /$ j.jnnfm.2006.11.003

[20] Folgar F., Tucker C. L.: Orientation behavior of fibers in concentrated suspensions. Journal of Reinforced Plastics and Composites, 3, 98-119 (1984).

DOI: $10.1177 / 073168448400300201$

[21] Kirkpatrick S.: Percolation and conduction. Reviews of Modern Physics, 45, 574-588 (1973).

DOI: $10.1103 /$ RevModPhys.45.574

[22] Sahimi M.: Flow phenomena in rocks: From continuum models to fractals, percolation, cellular automata, and simulated annealing. Reviews of Modern Physics, 65, 1393-1534 (1993). DOI: 10.1103/RevModPhys.65.1393

[23] Halperin B. I., Feng S., Sen P. N.: Differences between lattice and continuum percolation transport exponents. Physical Review Letters, 54, 2391-2394 (1985). DOI: $10.1103 /$ PhysRevLett.54.2391

[24] Feng S., Halperin B. I., Sen P. N.: Transport properties of continuum systems near the percolation threshold. Physical Review B, 35, 197-214 (1987).

DOI: 10.1103/PhysRevB.35.197

[25] Balberg I.: Limits on the continuum-percolation transport exponents. Physical Review B, 57, 13351-13354 (1998).

DOI: $10.1103 /$ PhysRevB.57.13351

[26] Skal A. S., Shklovskii B. I.: Topology of the infinite cluster of the percolation theory and its relationship to the theory of hopping conduction. Soviet Physics Semiconductors, 8, 1029-1032 (1975).

[27] de Gennes P. G.: On a relation between percolation theory and the elasticity of gels. Journal de Physique Lettres, 37, 1-2 (1976). DOI: 10.1051/jphyslet:019760037010100

[28] Stinchcombe R. B.: Branching model for percolation theory and electrical conductivity. Journal of Physics C: Solid State Physics, 6, L1-L5 (1973). DOI: 10.1088/0022-3719/6/1/001

[29] Semeriyanov F., Saphiannikova M., Heinrich G.: Anisotropic generalization of Stinchcombe's solution for the conductivity of random resistor networks on a Bethe lattice. Journal of Physics A: Mathematical and Theoretical, 42, 465001/1-465001/17 (2009). DOI: $10.1088 / 1751-8113 / 42 / 46 / 465001$ 
[30] Eken A. E., Tozzi E. J., Klingenberg D. J., Bauhofer W.: A simulation study on the combined effects of nanotube shape and shear flow on the electrical percolation thresholds of carbon nanotube/polymer composites. Journal of Applied Physics, 109, 084342/1-084342/9 (2011). DOI: $10.1063 / 1.3573668$

[31] Eken A. E., Tozzi E. J., Klingenberg D. J., Bauhofer W.: A simulation study on the effects of shear flow on the microstructure and electrical properties of carbon nanotube/polymer composites. Polymer, 52, 51785185 (2011). DOI: 10.1016/j.polymer.2011.08.041

[32] Schueler R., Petermann J., Schulte K., Wentzel H-P.: Percolation in carbon black filled epoxy resin. Macromolecular Symposia, 104, 261-268 (1996).

DOI: $10.1002 /$ masy. 19961040122

[33] Schueler R., Petermann J., Schulte K., Wentzel H-P.: Agglomeration and electrical percolation behavior of carbon black dispersed in epoxy resin. Journal of Applied Polymer Science, 63, 1741-1746 (1997).

DOI: $10.1002 /(\mathrm{SICI}) 1097-4628(19970328) 63: 13<1741$ $\because \mathrm{AID}-\mathrm{APP} 5>3.0 . \mathrm{CO} ; 2-\mathrm{G}$

[34] Heinrich G., Costa F. R., Abdel-Goad M., Wagenknecht U., Lauke B., Härtel V., Tschimmel J., Klüppel M., Svistkov A. L.: Structural kinetics in filled elastomers and PE/LDH composites. Kautschuk Gummi Kunstoffe, 58, 163-167 (2005).

[35] Skipa T., Lellinger D., Saphiannikova M., Alig I.: Shear-stimulated formation of multi-wall carbon nanotube networks in polymer melts. Physica Status Solidi B, 246, 2453-2456 (2009).

DOI: $10.1002 / \mathrm{pssb} .200982265$

[36] Skipa T., Lellinger D., Böhm W., Saphiannikova M., Alig I.: Influence of shear deformation on carbon nanotube networks in polycarbonate melts: Interplay between build-up and destruction of agglomerates. Polymer, 51, 201-210 (2010).

DOI: 10.1016/j.polymer.2009.11.047

[37] Alig I., Skipa T., Lellinger D., Bierdel M., Meyer H.: Dynamic percolation of carbon nanotube agglomerates in a polymer matrix: Comparison of different model approaches. Physica Status Solidi B, 245, 2264-2267 (2008).

DOI: $10.1002 / \mathrm{pssb} .200879622$

[38] Lellinger D., Xu D., Ohneiser A., Skipa T., Alig I.: Influence of the injection moulding conditions on the in-line measured electrical conductivity of polymercarbon nanotube composites. Physica Status Solidi B, 245, 2268-2271 (2008).

DOI: $10.1002 / \mathrm{pssb} .200879619$

[39] Torquato S.: Random heterogeneous materials: Microstructure and macroscopic properties, interdiciplinary applied mathematics. Springer, New York (2002).
[40] McLachlan D. S., Blaszkiewicz M., Newnham R. E.: Electrical resistivity of composites. Journal of the American Ceramic Society, 73, 2187-2203 (1990). DOI: $10.1111 / \mathrm{j} .1151-2916.1990 . t b 07576 . \mathrm{x}$

[41] Abbasi S., Carreau P. J., Derdouri A.: Flow induced orientation of multiwalled carbon nanotubes in polycarbonate nanocomposites: Rheology, conductivity and mechanical properties. Polymer, 51, 922-935 (2010). DOI: $10.1016 /$ j.polymer.2009.12.041

[42] Richter S., Saphiannikova M., Jehnichen D., Bierdel M., Heinrich G.: Experimental and theoretical studies of agglomeration effects in multi-walled carbon nanotube-polycarbonate melts. Express Polymer Letters, 3, 753-768 (2009).

DOI: $10.3144 /$ expresspolymlett.2009.94

[43] Kota A. K., Cipriano B. H., Powell D., Raghavan S. R., Bruck H. A.: Quantitative characterization of the formation of an interpenetrating phase composite in polystyrene from the percolation of multiwalled carbon nanotubes. Nanotechnology, 18, 505705/1505705/7 (2007).

DOI: $10.1088 / 0957-4484 / 18 / 50 / 505705$

[44] Cipriano B. H., Kota A. K., Gershon A. L., Laskowski C. J., Kashiwagi T., Bruck H. A., Raghavan S. R.: Conductivity enhancement of carbon nanotube and nanofiber-based polymer nanocomposites by melt annealing. Polymer, 49, 4846-4851 (2008).

DOI: $10.1016 /$ j.polymer.2008.08.057

[45] Huang Y. Y., Ahir S. V., Terentjev E. M.: Dispersion rheology of carbon nanotubes in a polymer matrix. Physical Review B, 73, 125422/1-125422/4 (2006). DOI: 10.1103/PhysRevB.73.125422

[46] Albano E. V., Mártin H. O.: Percolation of interacting particles deposited onto a planar substrate. Thin Solid Films, 151, 121-132 (1987). DOI: $10.1016 / 0040-6090(87) 90014-9$

[47] Seaton N. A., Glandt E. D.: Conductivity from simulations of percolating systems of interacting particles. Journal of Physics A: Mathematical and General, 20, 3029-3034 (1987). DOI: $10.1088 / 0305-4470 / 20 / 10 / 044$

[48] Dullaert K., Mewis J.: A structural kinetics model for thixotropy. Journal of Non-Newtonian Fluid Mechanics, 139, 21-30 (2006).

DOI: $10.1016 /$ j.jnnfm.2006.06.002

[49] Bilotti E., Zhang R., Deng H., Baxendale M., Peijs T.: Fabrication and property prediction of conductive and strain sensing TPU/CNT nanocomposite fibres. Journal of Materials Chemistry, 20, 9449-9455 (2010). DOI: $10.1039 / \mathrm{c} 0 \mathrm{jm} 01827 \mathrm{a}$

[50] Osswald T. A., Menges G.: Material science of polymers for engineers. Hanser, Munich (2003).

[51] Kharchenko S. B., Douglas J. F., Obrzut J., Grulke E. A., Migler K. B.: Flow-induced properties of nanotube-filled polymer materials. Nature Materials, 3, 564-568 (2004).

DOI: $10.1038 /$ nmat1183 
[52] Carreau P. J., De Kee D. C. R., Chhabra R. P.: Rheology of polymeric systems: Principles and applications. Hanser, New York (1997).

[53] Xu J., W, Florkowski W., Gerhardt R., Moon K-S., Wong C-P.: Shear modulated percolation in carbon nanotube composites. Journal of Physical Chemistry B, 110, 12289-12292 (2006).

DOI: $10.1021 / j p 061090 \mathrm{i}$
[54] Obrzut J., Douglas J. F., Kharchenko S. B., Migler K. B.: Shear-induced conductor-insulator transition in melt-mixed polypropylene-carbon nanotube dispersions. Physical Review B, 76, 195420/1-195420/9 (2007)

DOI: $10.1103 /$ PhysRevB.76.195420

[55] Friedman S. P., Seaton N. A.: Technical note: Percolation thresholds and conductivities of a uniaxial anisotropic simple-cubic lattice. Transport in Porous Media, 30, 241-250 (1998).

DOI: $10.1023 / \mathrm{A}: 1006575828107$ 